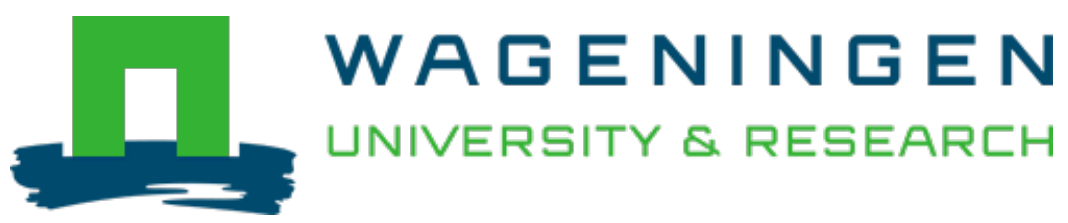

\author{
Influence of different proteolytic strains of Streptococcus thermophilus in co- \\ culture with Lactobacillus delbrueckii subsp. bulgaricus on the metabolite profile \\ of set-yoghurt \\ International Journal of Food Microbiology \\ Settachaimongkon, S.; Nout, M.J.R.; Antunes Fernandes, E.C.; Hettinga, K.A.; Vervoort, J.J.M. et al \\ https://doi.org/10.1016/j.ijfoodmicro.2014.02.008
}

This publication is made publicly available in the institutional repository of Wageningen University and Research, under the terms of article $25 \mathrm{fa}$ of the Dutch Copyright Act, also known as the Amendment Taverne. This has been done with explicit consent by the author.

Article 25 fa states that the author of a short scientific work funded either wholly or partially by Dutch public funds is entitled to make that work publicly available for no consideration following a reasonable period of time after the work was first published, provided that clear reference is made to the source of the first publication of the work.

This publication is distributed under The Association of Universities in the Netherlands (VSNU) 'Article $25 \mathrm{fa}$ implementation' project. In this project research outputs of researchers employed by Dutch Universities that comply with the legal requirements of Article $25 \mathrm{fa}$ of the Dutch Copyright Act are distributed online and free of cost or other barriers in institutional repositories. Research outputs are distributed six months after their first online publication in the original published version and with proper attribution to the source of the original publication.

You are permitted to download and use the publication for personal purposes. All rights remain with the author(s) and / or copyright owner(s) of this work. Any use of the publication or parts of it other than authorised under article $25 \mathrm{fa}$ of the Dutch Copyright act is prohibited. Wageningen University \& Research and the author(s) of this publication shall not be held responsible or liable for any damages resulting from your (re)use of this publication.

For questions regarding the public availability of this publication please contact openscience.library@wur.nl 


\title{
Influence of different proteolytic strains of Streptococcus thermophilus in co-culture with Lactobacillus delbrueckii subsp. bulgaricus on the metabolite profile of set-yoghurt
}

\author{
Sarn Settachaimongkon a,b,1, M.J. Robert Nout ${ }^{\mathrm{b}}$, Elsa C. Antunes Fernandes ${ }^{\mathrm{a}}$, Kasper A. Hettinga ${ }^{\mathrm{a}}$, \\ Jacques M. Vervoort ${ }^{\mathrm{c}}$, Toon C.M. van Hooijdonk ${ }^{\mathrm{a}}$, Marcel H. Zwietering ${ }^{\mathrm{b}}$, \\ Eddy J. Smid ${ }^{\text {b }}$, Hein J.F. van Valenberg ${ }^{\mathrm{a}, *}$ \\ a Dairy Science and Technology Group, Chair of Food Quality and Design, Wageningen University, P.O. Box 17, 6700 AA Wageningen, The Netherlands \\ b Laboratory of Food Microbiology, Wageningen University, P.O. Box 17, 6700 AA Wageningen, The Netherlands \\ c Laboratory of Biochemistry, Wageningen University, P.O. Box 8128, 6700 ET Wageningen, The Netherlands
}

\section{A R T I C L E I N F O}

\section{Article history:}

Received 25 July 2013

Received in revised form 17 January 2014

Accepted 14 February 2014

Available online 21 February 2014

\section{Keywords:}

Dairy

Starter culture

Fermented milk

Protease

Flavor

Metabolomics

\begin{abstract}
A B S T R A C T
Proto-cooperation between Streptococcus thermophilus and Lactobacillus delbrueckii subsp. bulgaricus is one of the key factors that determine the fermentation process and final quality of yoghurt. In this study, the interaction between different proteolytic strains of $S$. thermophilus and L. delbrueckii subsp. bulgaricus was investigated in terms of microbial growth, acidification and changes in the biochemical composition of milk during set-yoghurt fermentation. A complementary metabolomics approach was applied for global characterization of volatile and non-volatile polar metabolite profiles of yoghurt associated with proteolytic activity of the individual strains in the starter cultures. The results demonstrated that only non-proteolytic S. thermophilus (Prt - ) strain performed proto-cooperation with $L$. delbrueckii subsp. bulgaricus. The proto-cooperation resulted in significant higher populations of the two species, faster milk acidification, significant abundance of aroma volatiles and non-volatile metabolites desirable for a good organoleptic quality of yoghurt. Headspace SPME-GC/MS and ${ }^{1} \mathrm{H}$ NMR resulted in the identification of 35 volatiles and 43 non-volatile polar metabolites, respectively. Furthermore, multivariate statistical analysis allows discriminating set-yoghurts fermented by different types of starter cultures according to their metabolite profiles. Our finding underlines that selection of suitable strain combinations in yoghurt starters is important for achieving the best technological performance regarding the quality of product.
\end{abstract}

(c) 2014 Elsevier B.V. All rights reserved.

\section{Introduction}

Yoghurt is one of the most popular fermented dairy products and its consumption is increasing worldwide (Shiby and Mishra, 2013). According to the Codex standard for fermented milks (CODEX, 2003), yoghurt is specifically characterized by the presence of symbiotic cultures of Streptococcus thermophilus and Lactobacillus delbrueckii subsp. bulgaricus. During fermentation, these bacteria perform three major biochemical conversions of milk components: (i) conversion of lactose into lactic acid (fermentation), (ii) hydrolysis of caseins into peptides and free amino acids (proteolysis) and (iii) breakdown of milk fat into free fatty acids (lipolysis) (Smit et al., 2005). These reactions lead to the production of various metabolites resulting in a decrease of the $\mathrm{pH}$, formation of a semi-solid texture and a distinctive yoghurt flavor (Irigoyen et al., 2012). Even though S. thermophilus and L. delbrueckii

\footnotetext{
* Corresponding author. Tel.: + 31317 482284; fax: + 31317483669 .

E-mail address: hein.vanvalenberg@wur.nl (H.J.F. van Valenberg).

${ }^{1}$ Permanent address: Department of Food Technology, Faculty of Science, Chulalongkorn University, Bangkok 10330, Thailand.
}

subsp. bulgaricus are able to grow individually in milk, they can have a symbiotic interaction called "proto-cooperation" in mixed cultures (Smid and Lacroix, 2013). The interaction is based on the exchange of several metabolites which provide mutual growth stimulating effects (Sieuwerts et al., 2008). In summary, S. thermophilus produces pyruvic acid, formic acid, folic acid, ornithine, long-chain fatty acids and $\mathrm{CO}_{2}$ which stimulate the growth of $L$. delbrueckii subsp. bulgaricus. Lactic acid produced by $S$. thermophilus also reduces the $\mathrm{pH}$ of milk to an optimum level for L. delbrueckii subsp. bulgaricus. Consequently, the growth of $L$. delbrueckii subsp. bulgaricus supplies peptides, free amino acids and putrescine that stimulate the growth of $S$. thermophilus (Sieuwerts et al., 2008). Recent post-genomic studies in mixed culture of S. thermophilus and $L$. delbrueckii subsp. bulgaricus have revealed new insights in physiology and molecular basis of the interaction (Herve-Jimenez et al., 2009; Oliveira et al., 2012; Pastink et al., 2009; Sieuwerts et al., 2008; Sieuwerts et al., 2010). Although interaction between the two species is often positive (proto-cooperation), absence of interaction or even negative effects can take place depending on the combination of bacterial strains, type and heating process of base milk and fermentation conditions (Courtin and Rul, 2004). 
Proteolytic systems in lactic acid bacteria rely on the function of bacterial cell-envelope proteinases, peptide transport systems and intracellular peptidases (Mayo et al., 2010). Proteolytic activity of one of the species in the mixed cultures (mostly L. delbrueckii subsp. bulgaricus) plays an important role in proto-cooperation as mentioned previously. Traditional yoghurt cultures consist of non-proteolytic (Prt-) S. thermophilus and high proteolytic L. delbrueckii subsp. bulgaricus (Walstra et al., 2006). Thus, the former bacteria take advantage from active proteolytic system of the latter ones to meet their amino acid requirement. Furthermore, metabolism of amino acids generates volatile metabolites responsible for the aroma profile of product (Mayo et al., 2010). The contributions of $S$. thermophilus and L. delbrueckii subsp. bulgaricus to aroma volatile formation in fermented milk are well documented (Imhof and Bosset, 1994; Ott et al., 1997; Routray and Mishra, 2011). However, the expression of proteolytic activity (Prt +) in several S. thermophilus strains allows them to grow independently in milk leading to substantial acidification. This strong impact of proteolytic activity on acidifying capacity of $S$. thermophilus has been reported (Courtin et al., 2002; Dandoy et al., 2011; Galia et al., 2009). Despite extensive publications on the strain selections, mechanisms of amino acid biosynthesis, genome sequences and potential application of proteolytic $S$. thermophilus strains (Dandoy et al., 2011; Hols et al., 2005; Iyer et al., 2010), the role of these proteolytic streptococci in mixed culture with L. delbrueckii subsp. bulgaricus hardy received attention.

The developments in metabolomics allows discovery of a wide range of metabolites in complex biological systems including food matrices (Herrero et al., 2012). The application of mass spectrometry (MS) and nuclear magnetic resonance (NMR) technique have shown to be very successful in determining a wide range of metabolites related to microbial activity during fermentation, ripening and storage of fermented dairy products (Consonni and Cagliani, 2008; de Bok et al., 2011; Erkaya and Sengul, 2011; Harzia et al., 2012; Mozzi et al., 2013; Piras et al., 2013). However, to our knowledge, a complementary approach has never been applied to attain information regarding the effects of proteolytic activity of individual strains of $S$. thermophilus in mixed culture with L. delbrueckii subsp. bulgaricus during yoghurt fermentation on the global metabolite profile of product.

The objective of this study was therefore to investigate the interaction between proteolytic and non-proteolytic strains of $S$. thermophilus in mixed culture with $L$. delbrueckii subsp. bulgaricus during set-yoghurt fermentation. Growth of starter cultures, changes in milk pH and titratable acidity were monitored. Biochemical changes related to the interaction between the two species were characterized in terms of volatile and non-volatile polar metabolite profile of yoghurt using headspace SPME$\mathrm{GC} / \mathrm{MS}$ and ${ }^{1} \mathrm{H}$ NMR technique.

\section{Materials and methods}

\subsection{Milk preparation}

The milk was obtained by reconstituting $10 \%$ (w/v) Nilac skimmed milk powder (NIZO, Ede, The Netherlands) in milli-Q water $\left(45^{\circ} \mathrm{C}\right)$ to obtain final liquid milk at $9.5 \%$ dry matter content. The milk was pasteurized at $90{ }^{\circ} \mathrm{C}$ for $5 \mathrm{~min}$ and then was cooled down in a water bath until the temperature of $42{ }^{\circ} \mathrm{C}$ was reached.

\subsection{Starter cultures}

Frozen direct-vat-inoculation pellets of $S$. thermophilus Prt + (ST-Prt +) strain C38, S. thermophilus Prt - (ST-Prt -) strain C44 and L. delbrueckii subsp. bulgaricus Prt + (LB) strain C49 were supplied by CSK Food Enrichment (Ede, The Netherlands). The difference in proteolytic activity between the two $S$. thermophilus strains refers to the extracellular protease PrtS targeting milk proteins during yoghurt production. The pellets were stored at $-45^{\circ} \mathrm{C}$ and were placed at ambient temperature $\left(20 \pm 3{ }^{\circ} \mathrm{C}\right)$ for 15 min before use. Inoculation was performed to obtain an initial viable bacteria level at $10^{6} \mathrm{cfu} / \mathrm{g}$. Five different types of single strain and mixed cultures: (i) pure ST-Prt +, (ii) pure ST-Prt -, (iii) pure LB, (iv) mixed ST-Prt +/LB and (v) mixed ST-Prt - /LB were investigated in this study. The combinations of S. thermophilus and L. delbrueckii subsp. bulgaricus were inoculated at the ratio $10^{6}: 10^{6} \mathrm{cfu} / \mathrm{g}$ because in our previous experiments, this ratio had demonstrated the best profile (bacterial growth, acidity and texture) for yoghurt (data not shown).

\subsection{Set-yoghurt fermentation}

After inoculation, set-yoghurt fermentation was carried out in a water bath at $42^{\circ} \mathrm{C}$ for $4 \mathrm{~h}$. Samples were taken every hour during fermentation for microbiological and chemical analysis. The enumeration of viable bacteria and determination of $\mathrm{pH}$ and titratable acidity were carried out directly after sampling. For ${ }^{1} \mathrm{H}$ NMR analysis, the samples were stored at $-20{ }^{\circ} \mathrm{C}$ until the analysis. The fermentation was performed in three replicates for each type of starter culture.

\subsection{Enumeration of viable bacteria}

Viable bacteria in set-yoghurt were enumerated using the standard pour plate technique. Viable counts of $S$. thermophilus were determined on S. thermophilus agar after aerobic incubation at $37^{\circ} \mathrm{C}$ for $24 \mathrm{~h}$ (Ashraf and Shah, 2011). Viable counts of $L$. delbrueckii subsp. bulgaricus were determined on MRS agar pH 5.70 (Merck, Darmstadt, Germany) after anaerobic incubation (Anoxomat ${ }^{\mathrm{TM}}$-Mart ${ }^{\circledR}$ Microbiology, Drachten, the Netherlands) at $37^{\circ} \mathrm{C}$ for $48 \mathrm{~h}$ (Ashraf and Shah, 2011).

\subsection{Determination of acidification profile}

Production of acid during fermentation was expressed by changes in $\mathrm{pH}$ and increases in titratable acidity. Yoghurt samples were weighed to $25.0 \mathrm{~g}$ and the $\mathrm{pH}$ measurements were performed using a laboratory $\mathrm{pH}$ meter (InoLab pH720, WTW, Weilheim, Germany). The samples were titrated with $0.1 \mathrm{~N} \mathrm{NaOH}$ (Merck, Darmstadt, Germany) with continuous magnetic stirring until pH 8.30 was reached. The amount of $0.1 \mathrm{~N}$ $\mathrm{NaOH}(\mathrm{mL})$ used to titrate $100 \mathrm{~g}$ of yoghurt was referred as Titratable acidity (TA). The TA value was expressed as \% acid equivalent to lactic acid (\% LA) in the sample (ISO/IDF, 2009).

\subsection{Analysis of volatile metabolites by headspace SPME-GC/MS}

For headspace SPME-GC/MS analysis, a mimic-scenario of setyoghurt fermentation was carried out directly in GC vials to avoid loss of these compounds during sample preparation. The inoculated NILAC milk was directly divided ( $3 \mathrm{~mL}$ ) into a series of five clear headspace $\mathrm{GC}$ vials $(10 \mathrm{~mL}, 46 \times 22.5 \mathrm{~mm})$ sealed with $20 \mathrm{~mm}$ silicone/PTFE septa and magnetic caps (Grace, Albany, OR, USA). The vials were placed in a water bath at $42^{\circ} \mathrm{C}$ for $4 \mathrm{~h}$. The samples were stored at $-20^{\circ} \mathrm{C}$ until the analysis. In order to ensure that the results were comparable with the yoghurt fermented in Section 2.3, the final pH ( $4 \mathrm{~h}$ ) from in-vial fermentation was regularly verified (data not shown). The fermentation was performed in three replicates for each type of starter culture.

\subsubsection{Extraction of volatile compounds by Solid Phase Micro Extraction (SPME)}

Frozen samples were thawed and incubated at $60^{\circ} \mathrm{C}$ for $1 \mathrm{~min}$. Afterward, volatile compounds in the headspace were extracted at $60{ }^{\circ} \mathrm{C}$ for 5 min with a $75 \mu \mathrm{m}$ Carboxen ${ }^{\mathrm{TM}}$-PDMS-SPME fiber (Supelco, Bellefonte, PA, USA) using TriPlus ${ }^{\mathrm{TM}}$ autosampler (Thermo Scientific, Austin, TX, USA). Milli-Q water was analyzed as blank sample. This method was based on the method developed by Hettinga et al. (2008). 
2.6.2. Determination of volatile compounds by gas chromatography coupled with mass spectrometry (GC/MS)

The SPME fiber was desorbed for $10 \mathrm{~min}$ in the GC injection port. The GC/MS analysis was performed using Trace GC Ultra connected with DSQ II mass spectrometer (Thermo Scientific, Austin, TX, USA). The Stabilwax ${ }^{\circledR}$-DA-Crossband ${ }^{\circledR}-$ Carbowax ${ }^{\circledR}$-polyethylene-glycol column with $30 \mathrm{~m}$ length, $0.32 \mathrm{~mm}$ internal diameter, and $1 \mu \mathrm{m}$ film thickness (Restek, Bellefonte, PA, USA) was used. The oven temperature was maintained at $40{ }^{\circ} \mathrm{C}$ for $3 \mathrm{~min}$, then increased at $15{ }^{\circ} \mathrm{C} / \mathrm{min}$ to $220^{\circ} \mathrm{C}$ and maintained for $1 \mathrm{~min}$. The carrier gas was helium fed with a constant flow rate at $1.5 \mathrm{~mL} / \mathrm{min}$. The MS iron source was maintained at $225{ }^{\circ} \mathrm{C}$ with full scan. Electron impact mode was at $70 \mathrm{eV}$ with the mass range $33-250 \mathrm{~m} / \mathrm{z}$. This procedure was modified based on Hettinga et al. (2008). Volatile metabolites were identified using AMDIS software (NIST, Gaithersburg, MD, USA) referred to NIST/EPA/ NIH database and library provided by Hettinga et al. (2009). Peaks from column bleed and SPME fiber were corrected using the blank sample. Specific retention time and $\mathrm{m} / \mathrm{z}$ model were used for automated peak integration in XCalibur software package (Thermo Scientific, Austin, TX, USA).

\subsection{Analysis of non-volatile polar metabolites by ${ }^{1} H$ NMR spectroscopy}

\subsubsection{Sample preparation and ${ }^{1} H$ NMR analysis}

For ${ }^{1} \mathrm{H}$ NMR analysis, the samples from two replicates were analyzed. Frozen yoghurt samples were thawed at room temperature and $\mathrm{pH}$ was adjusted to 6.0 using $1.0 \mathrm{~N} \mathrm{NaOH}$ to achieve low variation, i.e. location and shape of peaks, in the spectra obtained (Mercier et al., 2011). Residual lipids were removed by dichloromethane extraction. The samples were diluted 1:2 (w/w) with dichloromethane (Merck, Darmstadt, Germany), then were mixed and centrifuged (Multifuge X3R, Thermo Scientific, Austin, TX, USA) at $4100 \mathrm{~g}$ for $15 \mathrm{~min}$ at $4{ }^{\circ} \mathrm{C}$. The clear liquid was collected and ultra-centrifuged (Beckman L60 Ultracentrifuge, Boulevard Brea, CA, USA) at 117,500 $\mathrm{g}$ for $75 \mathrm{~min}$ at $4{ }^{\circ} \mathrm{C}$ to remove the protein fraction. The clear serum was collected and ultra-filtrated using microcentrifugation (Spectrafuge ${ }^{\mathrm{TM}} 16 \mathrm{M}$ Microcentrifuge, Labnet Int. Inc., Woodbridge, NJ, USA) at $13,800 \mathrm{~g}$ for 20 min at room temperature through a Pall Nanosep ${ }^{\circledR}$ centrifugal device with $3 \mathrm{kDa}$ molecular weight cutoff (Pall life science, Ann Arbor, MI, USA). The filtrate was mixed $1: 1(\mathrm{v} / \mathrm{v})$ with phosphate buffer pH 6.0 (300 mM KH $\mathrm{HO}_{4}, 10 \%(\mathrm{w} / \mathrm{w}) \mathrm{D}_{2} \mathrm{O}$ and $1 \mathrm{mM}$ 3-(Trimethylsilyl) propionic-2,2,3,3-d4 acid sodium salt (TSP)) as internal standard. All chemicals used to prepare the buffer were obtained from SigmaAldrich (Steinheim, Germany). The mixture was stabilized at $4{ }^{\circ} \mathrm{C}$ overnight and then re-centrifuged at $13,800 \mathrm{~g}$ for $20 \mathrm{~min}$ at room temperature for final precipitate removal. Finally, $350 \mu \mathrm{L}$ of the mixture was transferred into a $4.25 \mathrm{~mm}$ NMR tube. NOESY $1 \mathrm{D}-{ }^{1} \mathrm{H}$ NMR measurements were performed at $300 \mathrm{~K}$ in a $600 \mathrm{MHz}$ NMR spectrometer (Bruker, Rheinstetten, Germany) equipped with a cryogenic probe, using Bruker sample handler (BACS-60) operated under full automation, with similar parameters as described by Lu et al. (2013).

\subsection{2. ${ }^{1} \mathrm{H}$ NMR spectra processing}

The ${ }^{1} \mathrm{H}$ NMR spectra were baseline-corrected, phase-corrected, aligned and calibrated based on the internal standard (TSP) peak. For each spectrum, chemical shift $(\delta)$ across the range of $0.00-10.00 \mathrm{ppm}$ was segmented (binning) with an interval of $0.02 \mathrm{ppm}$ (Anderson et al., 2011). The signal intensity in each bin was integrated and expressed in arbitrary units using AMIX software (Bruker, Rheinstetten, Germany). The bins corresponding with the water region $(\delta=4.73-$ $4.99 \mathrm{ppm})$ and methanol $(\delta=3.35-3.37 \mathrm{ppm})$ were eliminated from the analysis. Metabolite labels were presumptively assigned to the bins by means of Chenomx NMR suite 7.5 library (Chenomx Inc., Alberta, Canada), Human Metabolome Database version 3.0 (Wishart et al., 2013) and from literature (Boudonck et al., 2009; Klein et al., 2010; Lu et al., 2013). For unlabeled bins, significant variables were selected based on one-way ANOVA at 95\% confidence level.

\subsection{Statistical analysis}

One-way ANOVA with multiple comparisons by Tukey's test were performed using IBM SPSS statistics package version 19 (SPSS Inc., Chicago, IL, USA). A probability at $p<0.05$ was considered statistically significant. Metabolomics data from GC/MS and ${ }^{1} \mathrm{H}$ NMR were normalized by median-centering and $\log _{2}$-scaling before multivariate statistical analysis. Principle component analysis (PCA) and heat-map visualization combined with Pearson's correlation-based hierarchical cluster analysis (HCA) were performed using Multi-Experiment Viewer ( $\mathrm{MeV}$ ) version 4.8 (www.tm4.org/mev/).

\section{Results and discussion}

\subsection{Bacterial growth profiles}

The growth (increase in biomass) of ST-Prt + with LB (as pure and mixed culture) (Fig. 1A) and ST-Prt - with LB (as pure and mixed culture) (Fig. 1B) were monitored during fermentation. In pure cultures,
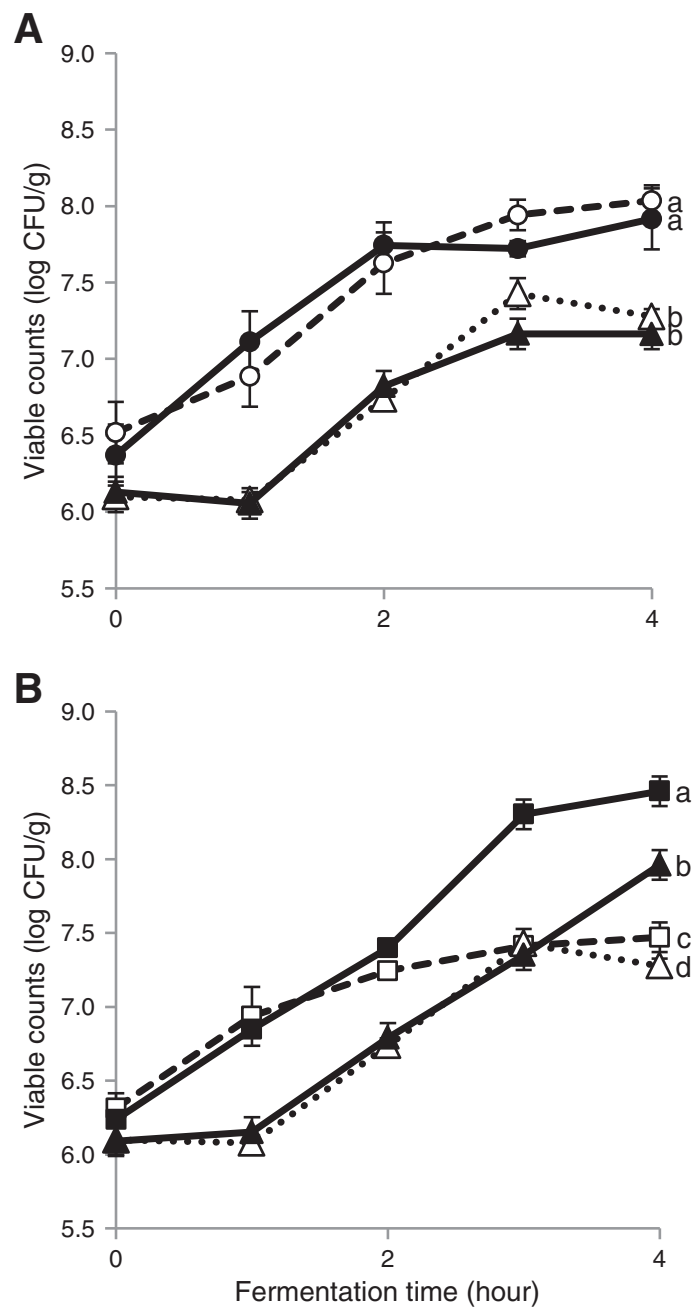

Fig. 1. Changes in viable counts during set-yoghurt fermentation with a pure culture of S. thermophilus Prt $+(-\infty$., panel A), pure culture of L. delbrueckii subsp. bulgaricus $(\ldots \wedge . .$, panel A) and their mixed culture $(\longrightarrow$, panel A) compared with pure culture of $S$. thermophilus Prt - (-n--, panel B), pure culture of L. delbrueckii subsp. bulgaricus $(\ldots, \ldots$, panel B) and their mixed culture $(\rightarrow-, \longrightarrow$ panel B). Error bars represent standard deviations based on three independent replicates. Letters $(a-c)$ indicate significant differences among mean values $(p<0.05)$ of samples at the end of fermentation $(4 \mathrm{~h})$. 
the two ST strains grew rapidly during the early part of fermentation $(0-3 \mathrm{~h})$, while LB remained in the lag-period for at least $1 \mathrm{~h}$. This can be explained by the initial $\mathrm{pH}$ of Nilac milk $(6.5 \pm 0.1)$ which is more favorable for the growth of ST (Walstra et al., 2006) and their effective capacity to use nutrients available in milk. These bacteria initially utilize free amino acids and peptides available in milk. However, the free nitrogen content in milk is very limited, usually not exceeding $100 \mathrm{mg} / \mathrm{L}$ depending on the animal breed, milking season, heat-treatment and storage (Letort and Juillard, 2001), thus only the ST-Prt + is expected to be able to continue growing with support of its proteolytic activity. Even though the LB in this study is a proteolytic strain, its growth was found to be retarded by a lower optimum $\mathrm{pH}$ and higher nutritional requirements (Sieuwerts et al., 2008). At the end of fermentation, the viable counts of pure ST-Prt + were significantly higher $(p<0.05)$ than those of pure ST-Prt- and pure LB which were not significantly different from each other. The final numbers increased by an average of $1.5 \mathrm{log}$ $\mathrm{cfu} / \mathrm{g}$ for pure ST-Prt + and $1.2 \mathrm{log} \mathrm{cfu} / \mathrm{g}$ for pure ST-Prt - and for pure LB. These results demonstrate that the ST-Prt + strain exhibited a significant higher capacity to develop individually in milk compared to ST-Prt - and LB. This observation is in agreement with the work of Courtin et al. (2002).

In mixed culture with LB, growth of the two ST strains started deviating from each other after $2 \mathrm{~h}$. The viable counts of ST-Prt + remained virtually constant towards the end of fermentation whereas those of STPrt - strongly increased ( $2-3 \mathrm{~h})$, with an average of $0.9 \mathrm{log} \mathrm{cfu} / \mathrm{g}$ before remaining at a stable level. The results suggested that ST-Prt - took advantage of the proteolytic activity of LB whereas ST-Prt + did not benefit from this interaction. At the end of fermentation, the viable counts of STPrt + in mixed culture were not significantly different $(p>0.05)$ from the pure culture. In contrast, the viable counts of ST-Prt - were significantly higher $(p<0.05)$ from those of the pure culture. The final numbers of ST-Prt + and ST-Prt - increased by an average of 1.5 and 2.3 $\log \mathrm{cfu} / \mathrm{g}$, respectively. The growth of LB in mixed culture with either ST-Prt + or ST-Prt - took place during 1-3 h. However, during 3-4 h, the viable counts of LB were constant in mixed culture with ST-Prt + while the counts in mixed culture with ST-Prt - still increased. This could be related to the continuous growth of ST-Prt - which consequently produces acid and lowers the $\mathrm{pH}$ to a level which favors for the growth of LB. Moreover, the LB might be stimulated by several metabolites produced by ST-Prt - as mentioned previously (Sieuwerts et al., 2008). At the end of fermentation, the viable counts of LB in pure culture and in mixed culture with ST-Prt + were not significantly different $(p>0.05)$ whereas its counts were significantly higher $(p<0.05)$ in mixed culture with ST-Prt - . The final numbers of LB in mixed culture with ST-Prt + and ST-Prt - increased by an average of 0.9 and $1.9 \log \mathrm{cfu} / \mathrm{g}$, respectively. This observation clearly demonstrates the proto-cooperation between ST-Prt - and LB resulting in significant higher populations of the two species at the end of fermentation. This mutual growth stimulation between ST and LB is in agreement with the results of Courtin and Rul (2004) and Herve-Jimenez et al. (2009). However, our results contrast with the findings of Courtin et al. (2002) who mentioned that the proteolytic activity of ST has no significant effect either on bacterial growth or final $\mathrm{pH}$ of yoghurt in mixed culture with a proteolytic LB. This might be explained by the differences in bacterial strains employed leading to their particular proteolytic profiles and other experimental factors such as type and pre-treatment of base milk as well as fermentation conditions.

\subsection{Acidification profiles}

The changes in $\mathrm{pH}$ were monitored every hour during fermentation (Fig. 2A). At the end of fermentation, the $\mathrm{pH}$ values of all yoghurts fermented by different types of starter cultures were significantly different $(p<0.05)$. Mixed ST-Prt $-/$ LB showed the best capacity to acidify milk followed by mixed ST-Prt +/LB, pure ST-Prt +, pure ST-Prt - and pure $\mathrm{LB}$ respectively. The two mixed cultures were able to acidify milk
A

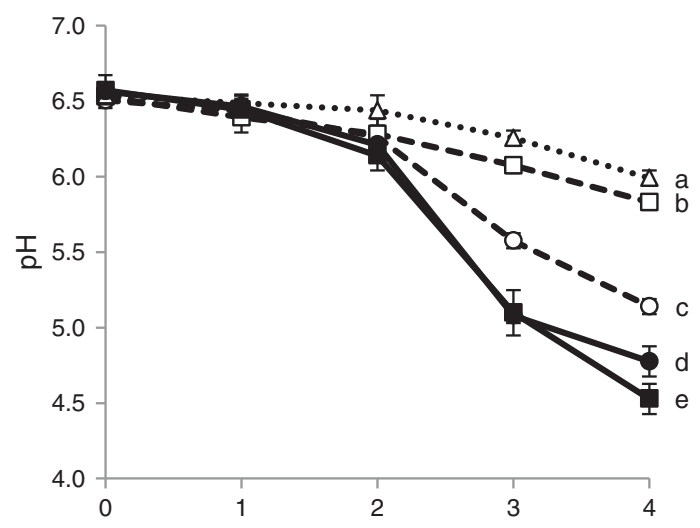

B

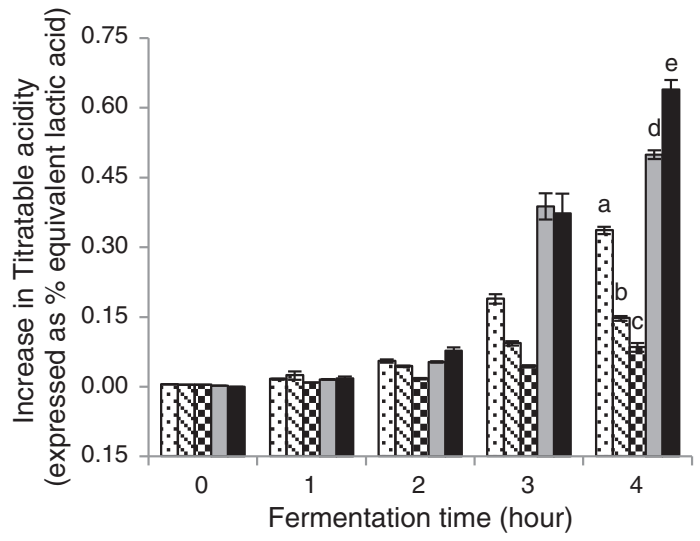

Fig. 2. Changes in pH (panel A) and titratable acidity (panel B) during set-yoghurt fermentation by a pure culture of $S$. thermophilus $\operatorname{Prt}+(\mathrm{O}, \mathrm{O})$, pure culture of $S$. thermophilus protease Prt $-(\square$, $\mathbb{S})$, pure culture of $L$. delbrueckii subsp. bulgaricus $(\Delta, \mathbb{R})$, mixed culture of S. thermophilus Prt + with L. delbrueckii subsp. bulgaricus $(\bullet, \square)$ and mixed culture of S. thermophilus Prt - with L. delbrueckii subsp. bulgaricus ( $\mathbf{\square}, \mathbf{\square})$. Error bars represent standard deviations based on three independent replicates. Letters (a-e) indicate significant differences among mean values $(p<0.05)$ of samples at the end of fermentation $(4 \mathrm{~h})$.

to a $\mathrm{pH}$ value below 5.0. However, only mixed ST-Prt -/LB was efficient in lowering $\mathrm{pH}$ to a value below 4.6 at which caseins aggregate (Walstra et al., 2006). Gel formation was only observed in the samples fermented with this mixed culture (data not shown). The similar capacity to lower milk pH by a mixed culture of ST and LB has also been reported by others (Courtin et al., 2002; Herve-Jimenez et al., 2009; Pinto et al., 2009).

Titratable acidity was expressed as \% acid equivalent to lactic acid (Fig. 2B). In pure cultures, ST-Prt + showed the best capacity to produce acid with an amount that is two-times higher than ST-Prt - and fourtimes higher than LB at the end of fermentation. In mixed culture, STPrt + did not show good interaction with LB. The amount of acid produced by mixed ST-Prt +/LB $(0.50 \%)$ was slightly higher than the sum of acid produced by pure ST-Prt + and pure LB together $(0.41 \%)$. This result can be associated with the populations of ST-Prt + and LB which are not significantly different in pure culture and mixed culture. On the other hand, the amount of acid produced by mixed ST-Prt-/LB (0.64\%) was almost three-times higher than the sum of acid produced by pure ST-Prt - and pure LB together $(0.22 \%)$. This observation is in accordance with the proto-cooperation observed on the growth of STPrt - and LB in mixed culture. Obviously, the significant higher populations of the two species are expected to lead to higher acid production.

\subsection{Volatile metabolite profiles determined by headspace SPME-GC/MS}

In this study, a total of 35 compounds consisting of alcohols, carbonyl compounds, organic acids, sulfur compounds and heterocyclic compound were identified in Nilac milk and set-yoghurts (Table S1). This 
list is comparable to the volatiles identified in yoghurt using headspace SPME-GC/MS in other studies (Condurso et al., 2008; Erkaya and Sengul, 2011). The 35 compounds were introduced as variables for multivariate analysis. If necessary, missing values were replaced by the median of respective variables.

An overall PCA score plot was constructed with a total variance of 73.4\% (Fig. 3A). The samples fermented with mixed ST-Prt -/LB were clearly separated from Nilac milk and from the samples fermented with pure LB along PC1 (51.9\% variance) while the distinction among the samples fermented with pure ST-Prt +, pure ST-Prt - and mixed ST-Prt +/LB was not clearly visible. PC1 loading indicated that acetaldehyde, diacetyl, acetoin, acetic acid and butyric acid mainly accounted for the separation of mixed ST-Prt -/LB from Nilac milk and other yoghurt samples.

Heat-map visualization combined with HCA (Fig. 4) demonstrated that acetaldehyde, dimethyl sulfide, 2-butanone, diacetyl, 2,3pentanedione, acetoin, 3-pentanol, 2-hydroxy-3-pentanone, acetic acid, butyric acid and hexanoic acid were present in high relative abundance (shown in red) in the samples fermented with mixed ST-Prt -/ LB. These compounds are desirable for a good organoleptic quality of yoghurt. The dendrogram showed that Nilac milk and different yoghurt samples could be well grouped according to their volatile metabolite profiles. Unlike PCA, the samples fermented with pure ST-Prt +, pure ST-Prt - and mixed ST-Prt +/LB could be clearly assigned into different clusters.

In terms of technological relevance, all major yoghurt aroma volatiles (Cheng, 2010); i.e. acetaldehyde (fresh, green, pungent), diacetyl (buttery, creamy), acetoin (buttery), 2-butanone (sweet, fruity), 2,3pentanedione (buttery, vanilla-like) and acetic acid (vinegar, pungent) were detectable in our study. The contributions of ST and LB to aroma volatile formation in fermented milk are well documented (Beshkova et al., 1998; Cheng, 2010; Tamime and Robinson, 2007; Walstra et al., 2006). In pure cultures, the total numbers of volatiles identified were almost equal in the samples fermented with pure ST-Prt $+(\mathrm{n}=20)$ and ST-Prt - $(\mathrm{n}=19)$ but higher compared to those in the samples fermented with pure LB $(n=16)$ (Table S1). Acetaldehyde is the most important compound contributing to typical yoghurt aroma which can be derived from amino acid catabolism (Cheng, 2010). In case of ST, threonine is converted into acetaldehyde by the activity of threonine aldolase (Iyer et al., 2010). The two pure ST cultures showed high capacity of acetaldehyde production without difference depending on their proteolytic activity. Indeed, the proteolytic activity of ST strains was expected to have an impact on the concentrations of various volatiles derived from amino acid catabolism; e.g. 1-methoxy-2-propanol (Val), 2-methyl-1-butanol (Ile/Leu), 3-methyl-3-butanol (Leu), benzaldehyde (Trp/Phe), 3-methyl-2-butenal (Ile/Leu), 2,3-pentanedione (Ile), 3-methyl-butanoic acid (Leu), 2-methyl-propanoic acid (Val), acetic acid (Thr) and sulfur compounds (Cys/Met) (Ardö, 2006; Smit et al., 2005). However, the two pure ST cultures only showed significant differences between each other in concentration of 2-methyl-1-butanol and 3-methyl-3-butanol. Possibly, the formation of these compounds by the two pure ST cultures initially relies on the utilization of free amino acids available in milk. Thus, the impact of different proteolytic activity between the two ST strains was not observed. In mixed cultures, although the total numbers of identified volatiles increased, relatively low numbers were found in the samples fermented with mixed STPrt $+/$ LB $(n=25)$ compared to mixed ST-Prt-/LB $(\mathrm{n}=32)$ (Table S1). It was apparent that mixed ST-Prt +/LB did not show a significant capacity to increase the concentration of major yoghurt aroma volatiles compared to their pure cultures. On the other hand, all major aroma volatiles were detected in significant higher abundance in the samples fermented with mixed ST-Prt - LB (Fig. 4). Furthermore, 3methyl-2-butanol, 3-octanone, 3-acetyl-2,5-dimethyl-furan, 3-methylbutanoic acid, 2-methyl-propanoic acid and pentanoic acid were exclusively detected in the samples fermented with this mixed culture. As previously mentioned, these compounds are derived from amino acid
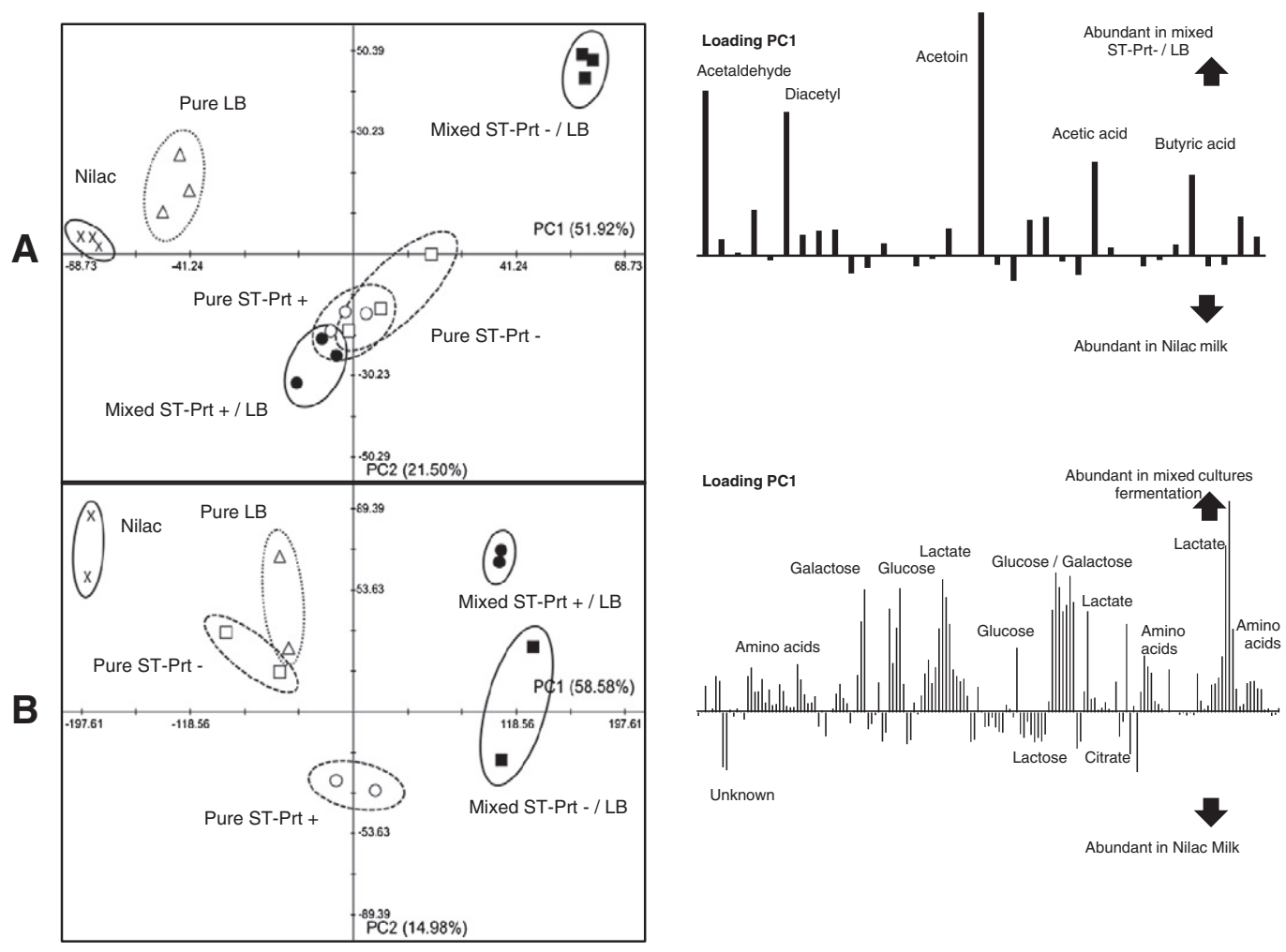

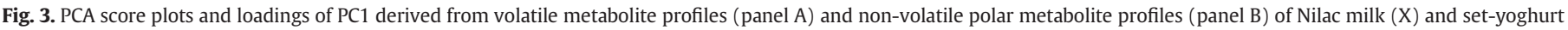

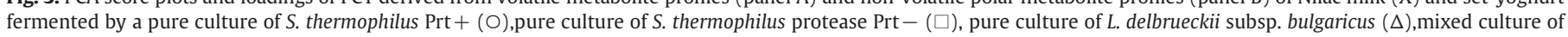
S. thermophilus Prt + with L. delbrueckii subsp. bulgaricus (-) and mixed culture of S. thermophilus Prt - with L. delbrueckii subsp. bulgaricus ( $\mathbf{\square}$ ). 


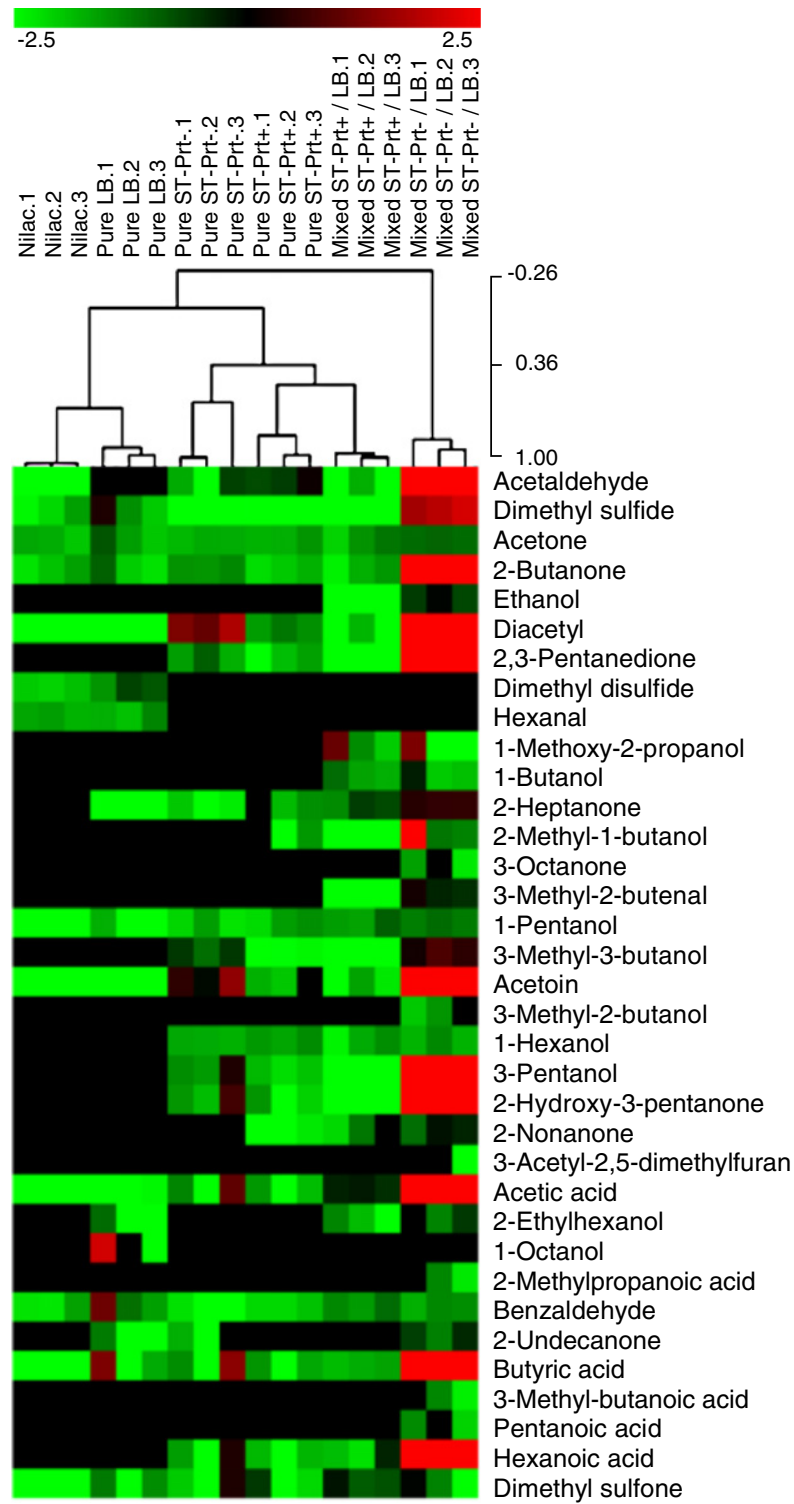

Fig. 4. Heat-map and hierarchical clustering of volatile metabolite profiles from Nilac milk and set-yoghurts fermented by different types of starter cultures. Dendrogram represents sample clusters based on Pearson's correlation coefficient with average linkage. Each square in the heat-map expresses normalized volatile content respected to the color range. The red color indicates higher content of the corresponding compound.

catabolism. Because the proteolytic activity of ST-Prt - is low, the formation of these compounds relies on proteolytic activity of LB in the mixed culture. Our results demonstrated that interaction between these two strains generated a favorable yoghurt volatile profile resulting in highest numbers of compounds identified with significant abundance of key-aroma compounds desirable for a good organoleptic quality of yoghurt. This finding confirms the proto-cooperation between STPrt - and LB as previously observed for bacterial growth and acidification profile.

\subsection{Non-volatile polar metabolite profiles determined by ${ }^{1} \mathrm{H} N \mathrm{NR}$}

In this study, a total of 43 metabolites including amino acids, carbohydrates, organic acids, lipid derivatives, carbonyl compounds, a sulfur compound and a nucleoside were presumptively identified (Fig. S1). The quantification was performed by summation of signal intensities in all bins corresponding to the target metabolite (Park et al., 2013).
The integrated intensities were finally expressed in $\log _{10}$ transformed (arbitrary unit) (Table S2). For multivariate analysis, it should be noted that the 43 identified metabolites accounted for labeling of 149 bins. A complementary data filtering by one-way ANOVA was performed for selection of the remaining unknowns (Lamanna et al., 2011). Finally, a total of 165 bins were introduced as variables for the analysis.

An overall PCA score plot was constructed with a total variance of 73.6\% (Fig. 3B). All yoghurt samples could be distinguished according to different types of starter cultures along PC1 (58.6\% variance). A complete distinction was observed between Nilac milk and yoghurts fermented with mixed cultures. The distinction between the samples fermented with pure ST-Prt - and pure LB was small but they could still be separated. Loading of PC1 indicated that lactose, citrate and unknown bins contributed to the separation of Nilac milk from fermented milk samples. The samples fermented with mixed cultures were clearly determined by lactate, glucose, galactose and most of the metabolites in aliphatic and aromatic region including organic acids and free amino acids. These compounds are well known as major products derived from milk fermentation (Tamime and Robinson, 2007; Zourari et al., 1992). Thus, the loading plot provides a good indication for metabolic activity of mixed cultures of ST and LB during set-yoghurt fermentation.

Heat-map visualization combined with HCA (Fig. 5) demonstrated that the majority of metabolites were present in high abundance (shown in red), with exceptions for citrate and lactose, in the samples fermented with mixed ST-Prt-/LB. Free amino acids were present in high relative abundance, especially in the samples fermented with pure ST-Prt + and mixed ST-Prt-/LB. Interestingly, these proteinbreakdown products were less present when ST-Prt + was inoculated in mixed culture with LB, although they are both proteolytic strains. The dendrogram showed that metabolite profiles of the samples fermented with pure LB and pure ST-Prt - were less different from Nilac milk, i.e. closely clustered together. This suggests lower metabolic activity of these two cultures during fermentation. Another main cluster consisted of the samples fermented with pure ST-Prt + which was well separated from mixed ST-Prt +/LB and mixed ST-Prt -/LB. It can be observed that Nilac milk and yoghurt samples are clearly grouped into different clusters according to their non-volatile polar metabolite profiles. This observation corresponds with the results obtained from PCA.

Changes in lactose, galactose and lactic acid concentrations in milk directly indicate the primary metabolic activity of ST and LB during yoghurt fermentation (Tamime and Robinson, 2007) (Table S2). In pure cultures, a significant decrease in lactose was only observed in the samples fermented with pure ST-Prt + . The concentration of lactate was significantly increased in the samples fermented with pure ST-Prt + followed by pure ST-Prt - and pure LB respectively. This observation agrees with the acidification profiles. In mixed cultures, the concentration of lactose and citrate significantly decreased while those of glucose, galactose and lactate were significantly increased. Moreover, dynamic changes in several organic acids, e.g. acetate, benzoate, citrate, formate, isobutyrate, orotate and succinate, were also revealed by ${ }^{1} \mathrm{H}$ NMR. It should be mentioned that these organic acids also contribute partially to the titratable acidy of yoghurt. The results demonstrate that mixed ST-Prt - /LB shows a higher capacity to generate these compounds compared to mixed ST-Prt +/LB.

The influence of proteolytic activity was characterized by an overall increase in free amino acid concentrations (proteolytic profile) (Table S2). In pure cultures, significant increases in N-acetyl amino acids, phenylalanine and valine were observed in the samples fermented with pure ST-Prt + whereas most of free amino acids were significantly decreased in the samples fermented with pure ST-Prt and pure LB. This result demonstrates the impact of different proteolytic activity between the two ST strains. Interestingly, the proteolytic profile of samples fermented with mixed ST-Prt +/LB was not significantly different from those observed in pure cultures, although both strains have an extracellular proteolytic activity. On the other hand, the proteolytic 

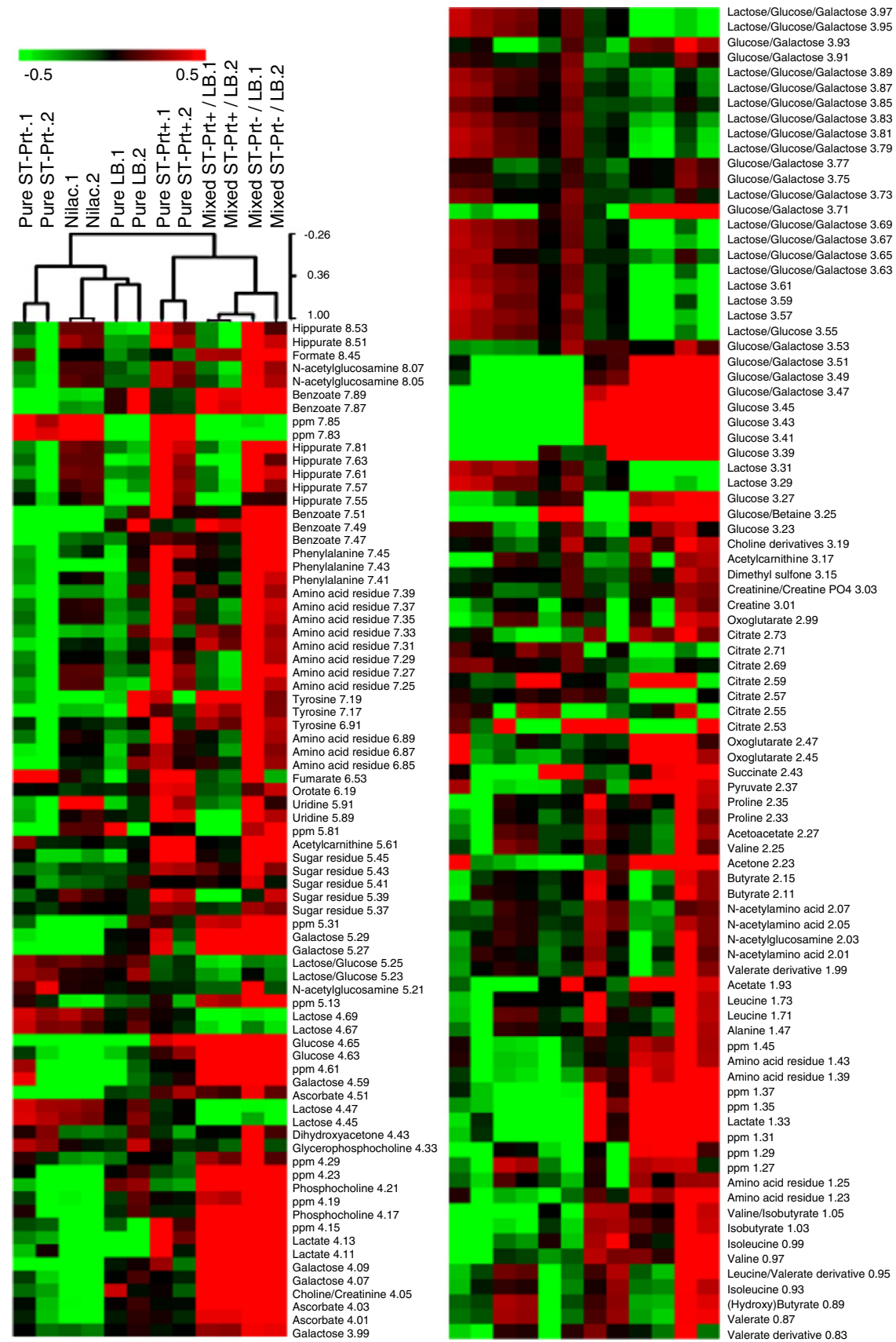

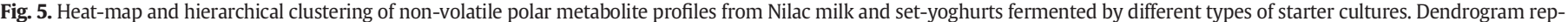

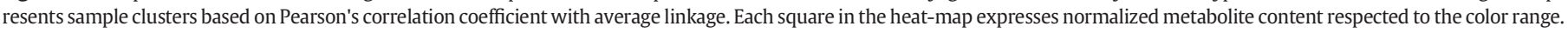
The red color indicates higher content of the corresponding compound.

profile of samples fermented with mixed ST-Prt -/LB was evidently increased. The concentrations of all free amino acids were significantly increased with an exception for tyrosine. The proto-cooperation between ST-Prt - and LB provides not only growth stimulatory effect on the two species but also exclusively stimulates the proteolytic activity of LB in mixed culture. This assumption is supported by the work of Sieuwerts et al. (2010) who reported a considerably higher expression of the proteolytic gene (prtB-LBUL-1105) responsible for the extracellular protease activity of LB in mixed culture.

In summary, the samples fermented with mixed ST-Prt -/LB demonstrated a significant higher level of non-volatile flavor compounds (Table S2), i.e. lactate, pyruvate, formate, succinate and free amino acids (as precursors for yoghurt aroma formation) for a good organoleptic quality of yoghurt (Cheng, 2010). These results confirm the proto-cooperation between ST-Prt - and LB as observed previously for bacterial growth, acidification and formation of aroma volatile compounds.

\section{Conclusions}

The present study demonstrated that selection of suitable strain combinations between $S$. thermophilus and L. delbrueckii subsp. bulgaricus in yoghurt starters is important for achieving the best technological performance regarding the quality of product. Although S. thermophilus Prt+ showed the best capacity to grow individually in milk, it did not interact well in mixed culture with L. delbrueckii subsp. bulgaricus. On the other 
hand, proto-cooperation between S. thermophilus Prt - and L. delbrueckii subsp. bulgaricus was evidently observed. The proto-cooperation resulted in significant higher populations of the two species, more efficient milk acidification, significant abundance of aroma volatiles and non-volatile metabolites desirable for a good organoleptic quality of yoghurt. A complementary metabolomics approach using headspace SPME-GC/MS and ${ }^{1} \mathrm{H}$ NMR resulted in the identification of 35 volatiles and 43 non-volatile polar metabolites, respectively. Furthermore, multivariate statistical analysis allows discriminating set-yoghurts fermented by different types of starter cultures according to their metabolite profiles.

\section{Acknowledgments}

This project was conducted under CHE-PhD-SFR-2551 scholarship granted by the Commission on Higher Education of the Royal Thai Government. Starter cultures were kindly provided by Bert Hafkamp from CSK Food Enrichment. The authors would like to acknowledge Geert Meijer for the GC/MS assistance. We also thank Bianca Hoopman, Katja Bobkova, Raôul Chin-A-Foeng, Anastasia Emelianova and Wu Qu for their technical contributions.

\section{Appendix A. Supplementary data}

Supplementary data to this article can be found online at http://dx. doi.org/10.1016/j.ijfoodmicro.2014.02.008.

\section{References}

Anderson, P.E., Mahle, D.A., Doom, T.E., Reo, N.V., Del Raso, N.J., Raymer, M.L., 2011. Dynamic adaptive binning: an improved quantification technique for NMR spectroscopic data. Metabolomics 7, 179-190.

Ardö, Y., 2006. Flavour formation by amino acid catabolism. Biotechnol. Adv. 24, 238-242.

Ashraf, R., Shah, N.P., 2011. Selective and differential enumerations of Lactobacillus delbrueckii subsp. bulgaricus, Streptococcus thermophilus, Lactobacillus acidophilus, Lactobacillus casei and Bifidobacterium spp. in yoghurt - a review. Int. J. Food Microbiol. 149, 194-208.

Beshkova, D., Simova, E., Frengova, G., Simov, Z., 1998. Production of flavour compounds by yogurt starter cultures. J. Ind. Microbiol. Biotechnol. 20, 180-186.

Boudonck, K., Mitchell, M., Wulff, J., Ryals, J., 2009. Characterization of the biochemical variability of bovine milk using metabolomics. Metabolomics 5, 375-386.

Cheng, H., 2010. Volatile flavor compounds in yogurt: a review. Crit. Rev. Food Sci. Nutr. $50,938-950$

CODEX, 2003. CODEX STAN 243-2003: standard for fermented milks [online]. Available at http://www.fao.org/docrep/015/i2085e/i2085e00.pdf (Accessed: November 3, 2012).

Condurso, C., Verzera, A., Romeo, V., Ziino, M., Conte, F., 2008. Solid-phase microextraction and gas chromatography mass spectrometry analysis of dairy product volatiles for the determination of shelf-life. Int. Dairy J. 18, 819-825.

Consonni, R., Cagliani, L.R., 2008. Ripening and geographical characterization of Parmigiano Reggiano cheese by ${ }^{1} \mathrm{H}$ NMR spectroscopy. Talanta 76, 200-205.

Courtin, P., Rul, F., 2004. Interactions between microorganisms in a simple ecosystem: yogurt bacteria as a study model. Lait 84, 125-134.

Courtin, P., Monnet, V., Rul, F., 2002. Cell-wall proteinases PrtS and PrtB have a different role in Streptococcus thermophilus/Lactobacillus bulgaricus mixed cultures in milk. Microbiology 148, 3413-3421.

Dandoy, D., Fremaux, C., Henry de Frahan, M., Horvath, P., Boyaval, P., Hols, P., Fontaine, L., 2011. The fast milk acidifying phenotype of Streptococcus thermophilus can be acquired by natural transformation of the genomic island encoding the cell-envelope proteinase PrtS. Microb. Cell Factories 10, S21.

de Bok, F.A.M., Janssen, P.W.M., Bayjanov, J.R., Sieuwerts, S., Lommen, A., van Hylckama Vlieg, J.E.T., Molenaar, D., 2011. Volatile compound fingerprinting of mixed-culture fermentations. Appl. Environ. Microbiol. 77, 6233-6239.

Erkaya, T., Sengul, M., 2011. Comparison of volatile compounds in yoghurts made from cows', buffaloes', ewes' and goats' milks. Int. J. Dairy Technol. 64, 240-246.

Galia, W., Perrin, C., Genay, M., Dary, A., 2009. Variability and molecular typing of Streptococcus thermophilus strains displaying different proteolytic and acidifying properties. Int. Dairy J. 19, 89-95.

Harzia, H. Kilk, K. Jõudu, I., Henno, M., Kärt, O., Soomets, U., 2012. Comparison of the metabolic profiles of noncoagulating and coagulating bovine milk. J. Dairy Sci. 95, 533-540.

Herrero, M., Simo, C., Garcia-Canas, V., Ibanez, E., Cifuentes, A., 2012. Foodomics: MS-based strategies in modern food science and nutrition. Mass Spectrom. Rev. 31, 49-69.

Herve-Jimenez, L., Guillouard, I., Guedon, E., Boudebbouze, S., Hols, P., Monnet, V., Maguin E., Rul, F., 2009. Postgenomic analysis of Streptococcus thermophilus cocultivated in milk with Lactobacillus delbrueckii subsp. bulgaricus: involvement of nitrogen, purine, and iron metabolism. Appl. Environ. Microbiol. 75, 2062-2073.
Hettinga, K.A., van Valenberg, H.J.F., Lam, T.J.G.M., van Hooijdonk, A.C.M., 2008. Detection of mastitis pathogens by analysis of volatile bacterial metabolites. J. Dairy Sci. 91, 3834-3839.

Hettinga, K.A., van Valenberg, H.J.F., Lam, T.J.G.M., van Hooijdonk, A.C.M., 2009. The origin of the volatile metabolites found in mastitis milk. Vet. Microbiol. 137, 384-387.

Hols, P., Hancy, F., Fontaine, L., Grossiord, B., Prozzi, D., Leblond-Bourget, N., Decaris, B., Bolotin, A., Delorme, C., Dusko Ehrlich, S., Guédon, E., Monnet, V., Renault, P. Kleerebezem, M., 2005. New insights in the molecular biology and physiology of Streptococcus thermophilus revealed by comparative genomics. FEMS Microbiol. Rev. 29, 435-463.

Imhof, R., Bosset, J.O., 1994. Relationships between micro-organisms and formation of aroma compounds in fermented dairy products. Z. Lebensm. Unters. Forsch. 198, 267-276.

Irigoyen, A., Ortigosa, M., Garcia, S., Ibanez, F.C., Torre, P., 2012. Comparison of free amino acids and volatile components in three fermented milks. Int. J. Dairy Technol. 65, 578-584.

ISO/IDF, 2009. ISO-PDTS11869/IDF-DRM150 Fermented Milks: Determination of Titratable Acidity - Potentiometric Method. International standard;ISO 14673-2International Organization for Standardization, Geneva.

Iyer, R., Tomar, S.K., Uma Maheswari, T., Singh, R., 2010. Streptococcus thermophilus strains: multifunctional lactic acid bacteria. Int. Dairy J. 20, 133-141.

Klein, M.S., Almstetter, M.F., Schlamberger, G., Nürnberger, N., Dettmer, K., Oefner, P.J. Meyer, H.H.D., Wiedemann, S., Gronwald, W., 2010. Nuclear magnetic resonance and mass spectrometry-based milk metabolomics in dairy cows during early and late lactation. J. Dairy Sci. 93, 1539-1550.

Lamanna, R., Braca, A., Di Paolo, E., Imparato, G., 2011. Identification of milk mixtures by ${ }^{1} \mathrm{H}$ NMR profiling. Magn. Reson. Chem. 49, S22-S26.

Letort, C., Juillard, V., 2001. Development of a minimal chemically-defined medium for the exponential growth of Streptococcus thermophilus. J. Appl. Microbiol. 91, 1023-1029.

Lu, J., Antunes Fernandes, E., Paez Cano, A.E., Vinitwatanakhun, J., Boeren, S., van Hooijdonk, T., van Knegsel, A., Vervoort, J., Hettinga, K.A., 2013. Changes in milk proteome and metabolome associated with dry period length, energy balance, and lactation stage in postparturient dairy cows. J. Proteome Res. 12, 3288-3296.

Mayo, B., Aleksandrzak-Piekarczyk, T., Fernandez, M., Magdalena, K., Alvarez-Martin, P., Bardowski, J., 2010. Updates in the mtabolism of lactic aid bcteria. In: Mozzi, F. Raya, R.R., Vignolo, G.M. (Eds.), Biotechnology of Lactic Acid Bacteria : Novel Applications. Wiley-Blackwell, Iowa, pp. 3-33.

Mercier, P., Lewis, M.J., Chang, D., Baker, D., Wishart, D.S., 2011. Towards automatic metabolomic profiling of high-resolution one-dimensional proton NMR spectra. J. Biomol. NMR 49, 307-323.

Mozzi, F., Ortiz, M.E., Bleckwedel, J., De Vuyst, L., Pescuma, M., 2013. Metabolomics as a tool for the comprehensive understanding of fermented and functional foods with lactic acid bacteria. Food Res. Int. 54, 1152-1161.

Oliveira, R.P.d.S., Torres, B.R., Perego, P., Oliveira, M.N.d., Converti, A., 2012. Co-metabolic models of Streptococcus thermophilus in co-culture with Lactobacillus bulgaricus or Lactobacillus acidophilus. Biochem. Eng. J. 62, 62-69.

Ott, A., Fay, L.B., Chaintreau, A., 1997. Determination and origin of the aroma impact compounds of yogurt flavor. J. Agric. Food Chem. 45, 850-858.

Park, S.J., Hyun, S.H., Suh, H.W., Lee, S.Y., Sung, G.H., Kim, S.H., Choi, H.K., 2013. Biochemical characterization of cultivated Cordyceps bassiana mycelia and fruiting bodies by 1H-nuclear magnetic resonance spectroscopy. Metabolomics 9, 236-246.

Pastink, M.I., Teusink, B., Hols, P., Visser, S., de Vos, W.M., Hugenholtz, J., 2009. Genomescale model of Streptococcus thermophilus LMG18311 for metabolic comparison of lactic acid bacteria. Appl. Environ. Microbiol. 75, 3627-3633.

Pinto, S.M., Clemente, M.D.G., De Abreu, L.R., 2009. Behaviour of volatile compounds during the shelf life of yoghurt. Int. J. Dairy Technol. 62, 215-223.

Piras, C., Cesare Marincola, F., Savorani, F., Engelsen, S.B., Cosentino, S., Viale, S., Pisano, M.B., 2013. A NMR metabolomics study of the ripening process of the Fiore Sardo cheese produced with autochthonous adjunct cultures. Food Chem. 141, 2137-2147.

Routray, W., Mishra, H.N., 2011. Scientific and technical aspects of yogurt aroma and taste: a review. Compr. Rev. Food Sci. Food Saf. 10, 208-220.

Shiby, V.K., Mishra, H.N., 2013. Fermented milks and milk products as functional foods: a review. Crit. Rev. Food Sci. Nutr. 53, 482-496.

Sieuwerts, S., de Bok, F.A.M., Hugenholtz, J., van Hylckama Vlieg, J.E.T., 2008. Unraveling microbial interactions in food fermentations: from classical to genomics approaches. Appl. Environ. Microbiol. 74, 4997-5007.

Sieuwerts, S., Molenaar, D., van Hijum, S.A.F.T., Beerthuyzen, M., Stevens, M.J.A., Janssen, P.W.M., Ingham, C.J., de Bok, F.A.M., de Vos, W.M., van Hylckama Vlieg, J.E.T., 2010 Mixed-culture transcriptome analysis reveals the molecular basis of mixed-culture growth in Streptococcus thermophilus and Lactobacillus bulgaricus. Appl. Environ. Microbiol. 76, 7775-7784.

Smid, E.J., Lacroix, C., 2013. Microbe-microbe interactions in mixed culture food fermentations. Curr. Opin. Biotechnol. 24, 148-154.

Smit, G., Smit, B.A., Engels, W.J.M., 2005. Flavour formation by lactic acid bacteria and biochemical flavour profiling of cheese products. FEMS Microbiol. Rev. 29, 591-610.

Tamime, A.Y., Robinson, R.K., 2007. Tamime and Robinson's Yoghurt: Science and Technology, 3rd ed. CRC Press, Cambridge.

Walstra, P., Wouters, J.T.M., Geurts, T.J., 2006. Dairy Science and Technology, 2nd ed. CRC/ Taylor \& Francis, New York.

Wishart, D.S., Jewison, T., Guo, A.C., Wilson, M., Knox, C., Liu, Y., Djoumbou, Y., Mandal, R. Aziat, F., Dong, E., Bouatra, S., Sinelnikov, I., Arndt, D., Xia, J., Liu, P., Yallou, F., Bjorndahl T., Perez-Pineiro, R., Eisner, R., Allen, F., Neveu, V., Greiner, R., Scalbert, A., 2013. HMDB 3.0: the human metabolome database in 2013. Nucleic Acids Res. 41, D801-D807.

Zourari, A., Accolas, J.P., Desmazeaud, M.J., 1992. Metabolism and biochemical characteristics of yogurt bacteria: a review. Lait 72,1-34. 